\title{
Voluntary apnea evokes diving responses in obstructive sleep apnea patients
}

\author{
Helena Vigetun-Haughey $\cdot$ Jonas Appelberg $•$ \\ Tomas Forsberg $•$ Magnus Kaldensjö • Erika Schagatay
}

Received: 1 July 2014 / Accepted: 13 December 2014 / Published online: 31 December 2014

(C) Springer-Verlag Berlin Heidelberg 2014

\begin{abstract}
Purpose Two potentially protective responses to apnea were studied in obstructive sleep apnea (OSA) patients; the diving response and the increase in $\mathrm{Hb}$ concentration $[\mathrm{Hb}]$ via spleen contraction.

Methods Eight OSA patients and ten healthy controls performed apneas in air (A) and apneas with facial immersion in $15{ }^{\circ} \mathrm{C}$ water (FIA) after inspiration and without prior hyperventilation. In each condition, subjects performed three apneas of maximal voluntary duration spaced by 2 min of rest. Cardiorespiratory parameters were measured
\end{abstract}

Communicated by Carsten Lundby.

H. Vigetun-Haughey $(\bowtie) \cdot$ T. Forsberg $\cdot$ M. Kaldensjö

E. Schagatay

Department of Health Sciences, Mid Sweden University,

Campus Östersund, 83125 Östersund, Sweden

e-mail: vigetun@gmail.com

E. Schagatay

e-mail: erika.schagatay@miun.se

H. Vigetun-Haughey

Department of Clinical Physiology, Capio St Görans Hospital,

Stockholm, Sweden

J. Appelberg

Department of Research and Development, Sundsvall Hospital,

Västernorrland County Council, Sundsvall, Sweden

e-mail: jonas.appelberg@1vn.se

\section{J. Appelberg}

Department of Clinical Physiology, Sundsvall Hospital,

Västernorrland County Council, Sundsvall, Sweden

E. Schagatay

Swedish Winter Sports Research Center, Mid Sweden University,

Östersund, Sweden non-invasively, and venous blood samples for [Hb] analysis were drawn before and after apneas.

Result Mean (SD) apnea durations were similar between groups (NS). In controls, the heart rate (HR) reduction was $10 \pm 10 \%$ at apnea and $19 \pm 10 \%$ in FIA $(P<0.05)$. In OSA patients, however, the fall in HR was the same in both conditions, $13 \pm 10$ and $14 \pm 8 \%$ for A and FIA, respectively (NS). In controls, the [Hb] increase was the same in A and FIA $(2.2 \pm 2.9$ and $2.1 \pm 2.2 \%)$, while in OSA the $[\mathrm{Hb}]$ increase was greater during FIA compared to A $(3.3 \pm 2.2$ and $1.4 \pm 0.9 \% ; P<0.05)$.

Conclusion Apnea induces a diving response and [Hb] increase in both groups. OSA patients did not show the typical training effect of the diving response seen in apnea divers despite their frequent nocturnal apneas. However, they also deviated from normal controls in response pattern; face immersion enhanced the cardiovascular diving response in controls but not in OSA, while the hematological response was enhanced by face immersion only in OSA patients.

Keywords Training - Spleen contraction - Hypoxia · Hypercapnia $\cdot \mathrm{Hb} \cdot$ Bradycardia $\cdot$ OSA $\cdot$ Hemoglobin

$\begin{array}{ll}\text { Abbreviations } \\ \text { A } & \text { Apnea without face immersion } \\ \text { AHI } & \text { Apnea/hyponea index } \\ \text { AT } & \text { Apneic time } \\ \text { BMI } & \text { Body mass index } \\ \text { EP } & \text { Easy phase } \\ { }_{\text {ET }} \mathrm{CO}_{2} & \text { Tension for end-tidal carbon dioxide } \\ \text { FIA } & \text { Apnea with face immersion } \\ \text { [Hb] } & \text { Blood concentration of hemoglobin } \\ \mathrm{HR} & \text { Heart rate } \\ \text { IBM } & \text { Involuntary breathing movements } \\ \text { MAP } & \text { Mean arterial blood pressure }\end{array}$




$\begin{array}{ll}\text { ODI } & \text { Oxygen desaturation index } \\ \text { OSA } & \text { Obstructive sleep apnea } \\ \mathrm{PaCO}_{2} & \text { Partial pressure carbon dioxide } \\ \mathrm{SaO}_{2} & \text { Blood hemoglobin oxygen saturation } \\ \mathrm{SD} & \text { Standard deviation } \\ \mathrm{SkBf} & \text { Skin blood flow }\end{array}$

\section{Introduction}

The human diving response

Patients with obstructive sleep apnea (OSA) are frequently exposed to involuntary obstructive apneas and hypoxia during sleep, which enhances the risk of cardiovascular disease. An obstructive apnea is typically accompanied by a reduction in heart rate (HR) (Zwillich et al. 1982). This reaction is most likely triggered by the concomitant hypoxia following the apnea (Daly and Hazzledine 1963), and is more pronounced during sleep in comparison to wakefulness (Tolle et al. 1983). During normal breathing, hypoxia will trigger a tachycardic reaction, but with a simultaneous breath hold, the opposite reaction in heart rate, a bradycardia, overrides the tachycardic reaction as part of the diving response (Gooden 1994). During voluntary apnea when awake, a series of cardiovascular reflexes collectively called the "diving response" is initiated to prevent hypoxia of oxygen dependent organs. The response is observed in many air-breathing divers among mammals, reptiles and birds, and characterized by peripheral vasoconstriction and a reduction of the HR (Butler and Jones 1997). This is a preventive response developing prior to hypoxia and occurs via a sympathetic input to peripheral vessels and by parasympathetic input on the heart (Gooden 1994). The response is also present in healthy humans, where it is induced mainly by voluntary apnea and fortified by facial chilling, via cold receptors in the upper part of the face (Schuitema and Holm 1988; Gooden 1994). It is clear that this response, which has been extensively studied in human apneic diving models, is protective during voluntary apneas in healthy humans, leading to prolonged apneas (Schagatay and Andersson 1998) or less arterial desaturation in a given apneic time (Andersson and Schagatay 1998a). The mechanisms responsible for oxygen conservation are both the vasoconstriction, directing the blood mainly to the brain and heart, while other organs may rely on anaerobic metabolism (Elsner and Gooden 1983) and the reduced work of the myocardium during bradycardia with subsequent reduction in its metabolism (Lin 1982).

\section{$\mathrm{Hb}$ elevation via spleen contraction}

The amount of circulating red blood cells in many diving mammals changes between diving and non-diving states due to contraction of the large, compliant spleen, which serves as an "external" blood storage (Qvist et al. 1986). The release of erythrocytes into the blood, as a result of spleen contraction during apneic diving, is also found in humans (Hurford et al. 1990) and may be a key factor in prolonging apneas with repetition (Schagatay et al. 2001; Bakovic et al. 2003). Simulated diving by apnea with face immersion, but also apnea alone, will trigger contraction of the spleen in humans, with the full response developing across three to four serial apneas (Bakovic et al. 2003; Schagatay et al. 2005). While hypoxia has been identified as an important input (Richardson et al. 2009), other factors such as hypercapnia related to the apneic event may also modify the response (Lodin-Sundstrom and Schagatay 2010).

Voluntary and involuntary apnea

When a voluntary apnea is initiated, it begins with a phase without respiratory movements and with no subjective urge to breathe, the "easy going phase" (EP); but when partial pressure for carbon dioxide $\left(\mathrm{PaCO}_{2}\right)$ rises, involuntary respiratory movements will be triggered at the physiological breaking point (Lin et al. 1974). Voluntary continuation of the apnea is often possible during the "struggle phase" following onset of involuntary breathing movements (IBM; Dejours 1965) until an individual breaking point, when the urge to breathe can no longer be voluntarily resisted (Lin et al. 1974). While the initial phase may resemble involuntary central apneas during sleep, the second phase involving progressively increasing thoracic movements (with pressure shifts due to inspiratory activity against a closed glottis) is more similar to OSAs. However, the diving bradycardia develops across the apnea with little influence from this phase shift (Andersson and Schagatay 1998b), or with a possible increased fall in HR toward the end of the apnea (Palada et al. 2008). Small lung volume may have some influence on the diving response via lung-stretch receptor input and possibly also by the magnitude of oxygen stores being smaller with a smaller lung volume, leading to faster desaturation (Andersson and Schagatay 1998b).

\section{Training of protective responses}

The general pattern of adaptations to apneic diving can be summarized in three main categories aiming to: (1) increase oxygen stores, (2) increase resistance to asphyxia, and (3) reduce metabolism (Schagatay 2009). Traininginduced enhancements of any of these factors would likely be protective in a variety of asphyxic situations. The diving response has been shown to be more pronounced in trained apnea divers (Schagatay and Andersson 1998), and long-term training studies have revealed a training effect 
(Schagatay et al. 2000; Engan et al. 2013). An increase in blood hemoglobin concentration ([Hb]) during apnea, as well as spleen volume changes, has been reported to be greater in elite apnea divers than in non-divers, possibly suggesting training-induced changes (Richardson et al. 2005; Schagatay et al. 2007). However, such inter-group differences could also be derived from pre-selection. A longitudinal study involving 2 weeks of apnea training did not demonstrate training effect on the spleen or $[\mathrm{Hb}]$ elevation (Engan et al. 2013), while a recent study on 2 months of high altitude hypoxia during a climb of Mt Everest did find an enhanced spleen contraction (Engan et al. 2014), suggesting that training effects may arise only after a longtime exposure to hypoxia. Therefore, the central aim was to study the $\mathrm{Hb}$ response development and consequences in voluntary apneas in OSA patients, as they have been frequently exposed to nocturnal apneas for a long period of time.

The main aim was to study the apnea-induced "diving response" and the spleen-derived $[\mathrm{Hb}]$ elevation in patients with OSA, and to clarify if these patients have enhanced responses compared to control non-divers. The questions addressed were if there are differences between groups with respect to apneic duration, onset of involuntary breathing movements, diving response (heart rate, blood pressure, and skin blood flow changes), effect of facial immersion on diving response parameters, arterial oxygen saturation after apneas, and hematological response to apneas.

\section{Methods}

\section{Subjects}

The study population consisted of seven men and one woman who had been referred to the Department of Clinical Physiology, Sundsvall Hospital, for investigation of snoring and daytime sleepiness. They were all diagnosed as having OSA by an ambulatory sleep apnea recording method (Embletta Portable Diagnostic System, Embla Systems, Bromfield, CO, USA) identifying an apnea/hypopnea index (AHI) exceeding 10 per hour (Dingli et al. 2003). In addition, a control group consisting of ten healthy volunteers matched for age, height, weight, and body mass index (BMI) were studied (Table 1). The volunteers were recruited among friends and colleagues at Mid Sweden University, Sweden. All subjects in the control group also underwent a nocturnal registration of apneas and desaturations during one night in their homes, ensuring they were free of OSA. None of the OSA patients had received treatment for OSA, reported a history of cardiovascular disease or were undergoing treatment with beta blockers. The study complied with the Helsinki Declaration, Swedish laws and
Table 1 Subject characteristics

\begin{tabular}{lll}
\hline & Controls $(n=10)$ & OSA $(n=8)$ \\
\hline Age (years) & $51.0 \pm 6.3$ & $53.0 \pm 9.9$ \\
Sex ratio (M/F) & $6 / 4$ & $7 / 1$ \\
Height $(\mathrm{cm})$ & $176.0 \pm 5.4$ & $175.4 \pm 8.2$ \\
Body weight $(\mathrm{kg})$ & $89.6 \pm 10.1$ & $90.3 \pm 15.5$ \\
BMI $\left(\mathrm{kg} / \mathrm{m}^{2}\right)$ & $29.0 \pm 3.2$ & $29.2 \pm 4.0$ \\
Vital capacity $(\mathrm{l})$ & $4.4 \pm 0.8$ & $4.2 \pm 0.9$ \\
AHI*** & $3.3 \pm 2.3$ & $47.7 \pm 16.2$ \\
ODI*** & $3.7 \pm 2.8$ & $45.5 \pm 23.7$ \\
Physical activity (h/week) & $3.1 \pm 2.4$ & $1.3 \pm 2.4$ \\
\hline
\end{tabular}

Values are group mean $\pm \mathrm{SD}$

$B M I$ body mass index, $A H I$ apnea/hypopnea index, $O D I$ oxygen desaturation index

*** $P \leq 0.001$

ethical standards and had been approved by the regional human ethics committee at Umeå University, Umeå, Sweden.

\section{Procedures}

Subjects arrived at the laboratory after $1 \mathrm{~h}$ free of food, tobacco, and caffeine. After receiving thorough information about the methods and signing an informed consent form, subjects underwent the following procedures. A catheter was inserted in a ante-cubital vein and the vital capacity was measured in the standing position with a spirometer (Vitalograph, Compact II, Buckingham, England) using the highest value of 3 measurements. The subjects then rested in horizontal position for $30 \mathrm{~min}$. The last $10 \mathrm{~min}$ were always in the prone position, and vital capacity was measured again in this position.

\section{Apnea protocol}

All subjects performed two series of three apneas at individual maximal duration: one series with the face immersed in water of $15{ }^{\circ} \mathrm{C}( \pm 0.2)$ (FIA) and the other in air (A). The subject's face was dried between immersions. The serial apneas were spaced by $2 \mathrm{~min}$ of quiet rest and normal breathing and the two series were separated by $20 \mathrm{~min}$ of rest in the prone position (Fig. 1). The order of the two different types of apneas was weighed. After application of a nose clip at $30 \mathrm{~s}$ prior to the apnea, a count down of $10 \mathrm{~s}$ was performed during which the subject was breathing through a mouthpiece connected to the gas analyzer. The subject was instructed to exhale fully through the mouthpiece and then to take a deep but not maximal breath before starting the apnea. This has previously been found to lead to a lung volume of approximately $85 \%$ of vital capacity (Schagatay 1996). At the end of voluntary maximal apnea 


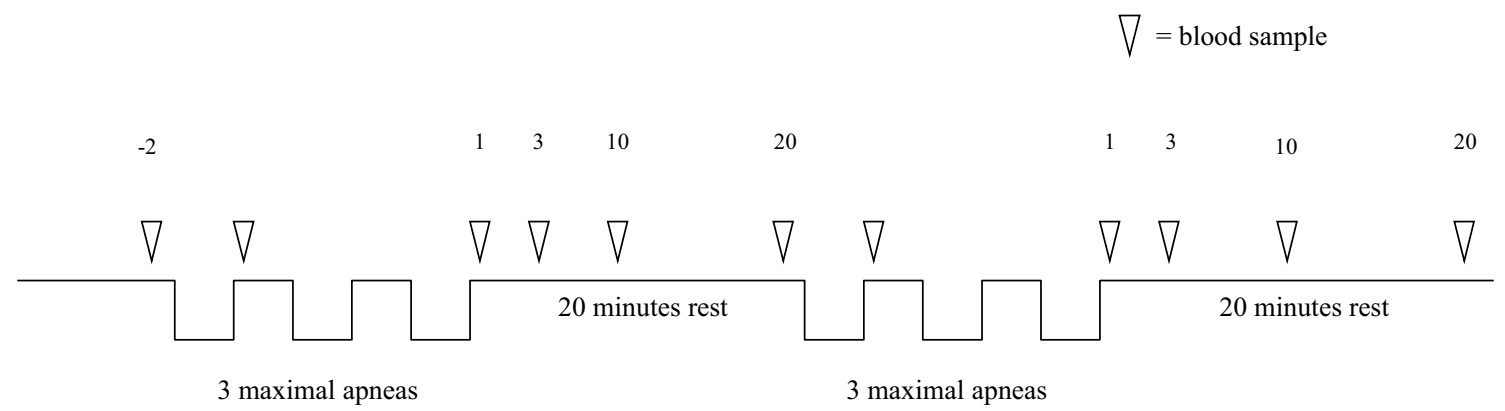

Fig. 1 Apnea protocol and blood sample timing during the experiment. The numbers above the figures indicate at what time, in reference to the apnea series, the blood samples were drawn. Two minutes before $(-2)$ and minutes after the apneas

duration, the subject exhaled through the mouthpiece and continued breathing at normal rate until next apnea started after countdown.

\section{Measurements}

Measurements were registered non-invasively from $2 \mathrm{~min}$ before until $2 \mathrm{~min}$ after the last apnea of each series. The following probes were attached to the subject's hand of the arm without the venous catheter; a cuff on the middle finger located at heart level was used for HR and mean arterial blood pressure (MAP) registration by photoplethysmography (Finapress, Ohmeda 2300, Ohmeda, Madison, WI, USA), a probe on the thumb for skin blood flow (SkBf) registration using laser Doppler technique (Laser Flowmeter 21, Advance, Tokyo, Japan), and capillary blood hemoglobin saturation $\left(\mathrm{SaO}_{2}\right)$ was measured using pulse oximetry on the forefinger (Pulsoxymeter 2800, Dansjö Medical, Bromma, Sweden). Respiratory movements were measured using a laboratory-developed pneumatic chest bellows connected to an amplifier. Endtidal $\mathrm{CO}_{2}\left({ }^{\mathrm{ET}} \mathrm{CO}_{2}\right)$ in breaths just prior to and directly after apnea was measured with a $\mathrm{CO}_{2} / \mathrm{O}_{2}$ analyzer (Normocap Oxy, Datex Ohmeda, Helsinki, Finland) connected to the mouthpiece of the spirometer. Cardiovascular data were stored via a Biopac system (Biopac Systems, Goleta, CA) using the computer program AcqKnowledge and analyzed in Microsoft Excel.

\section{Blood sampling}

Blood samples were drawn at 30,20, 10 and $2 \mathrm{~min}$ prior to starting the apnea protocol, directly after the first and directly after the third apnea and then 3, 10, and 20 min post apnea in both the series (Fig. 1). The total amount of blood drawn from each subject, including waste samples, was approximately $30 \mathrm{ml}$ and approximately $10 \mathrm{ml}$ of saline was inserted to rinse the catheter.

\section{Analysis}

Apnea duration and the duration of EP were determined, the latter by identifying the onset of IBM from the registration of thoracic movements; the first clear inspiratory effort was used as the EP breaking point. Subjects served as their own controls for changes obtained during apneas. Reference values for $\mathrm{HR}, \mathrm{SkBF}, \mathrm{SaO}_{2}$, and MAP were sampled as mean values during the last $30 \mathrm{~s}$ prior to the start of the first apnea in the series, and reference values for ${ }^{\mathrm{ET}} \mathrm{CO}_{2}$ were obtained from the last expiration before each apnea. The apnea values for MAP and SkBf used for comparing conditions and groups were mean values during the whole apnea. Heart rate values during the last $5 \mathrm{~s}$ of the apnea were used to avoid the initial tachycardia and HR decline. The $\mathrm{SaO}_{2}$ values used were the nadirs found in the period from apnea onset to $30 \mathrm{~s}$ post apnea. The blood samples were analyzed in triplicates for $[\mathrm{Hb}]$ with automated analysis equipment (Micros 60 Analyzer, ABX Diagnostics, Montpellier, France). Samples drawn 2 min prior to apneas were assigned as reference values.

The data are presented as mean \pm standard deviation (SD). All data were analyzed as group means, originating from individual means for the three attempts. Paired Student's $t$ test was used within groups and, for comparison between groups, unpaired Student's $t$ test was used. $P \leq 0.05$ was considered to be statistically significant.

\section{Results}

Apneic duration and involuntary breathing movements

Both types of apneas produced apneic time (AT) and EP of the same durations between groups and there was no significant difference when types of apneas were compared within groups. In controls, AT $(69 \pm 35 \mathrm{~s}$ for A, $50 \pm 24 \mathrm{~s}$ for FIA) was longer than $\mathrm{EP}(60 \pm 23 \mathrm{~s}$ for $\mathrm{A}, 54 \pm 21 \mathrm{~s}$ 
Table 2 Respiratory parameters for pre and post apnea

\begin{tabular}{lllll}
\hline & Pre-apnea & \\
& ${ }^{\mathrm{ET}} \mathrm{CO}_{2}(\%)$ & Post-apnea ${ }^{\mathrm{ET}} \mathrm{CO}_{2}(\%)$ & Pre-apnea $\mathrm{SaO}_{2}(\%)$ & $\mathrm{Post}_{-a p n e a ~} \mathrm{SaO}_{2}(\%)$ \\
\hline Controls A $(n=9)$ & $4.3 \pm 0.6$ & $6.0 \pm 0.7^{* * *}$ aa & $96.8 \pm 1.5$ & $94.5 \pm 2.1^{* *}$ \\
Controls FIA $(n=9)$ & $4.3 \pm 0.6$ & $5.9 \pm 0.6^{* * *}$ ff & $95.9 \pm 2.3$ & $94.8 \pm 0.9^{* *}$ \\
OSA A $(n=8)$ & $4.7 \pm 0.6$ & $5.7 \pm 0.4^{* * *}$ & $96.2 \pm 1.4$ & $94.6 \pm 1.5^{* *}$ \\
OSA FIA $(n=8)$ & $4.7 \pm 0.6$ & $5.5 \pm 0.6^{* * *}$ & $96.4 \pm 1.4$ & $94.1 \pm 2.3^{* *}$ \\
\hline
\end{tabular}

Values are group mean $\pm \mathrm{SD}$

$* P \leq 0.05 * * P \leq 0.01 * * * P \leq 0.001$

aa indicating difference between controls A compared to OSA; $P \leq 0.01$

ff indicating difference between controls FIA compared to OSA FIA; $P \leq 0.01$

for FIA), thus a struggle phase was seen in both types of apneas $(P<0.05)$. OSA patients, however, often terminated their apnea at the end of EP, at a mean duration of $49 \pm 35 \mathrm{~s}$ for A. In FIA, however, AT was slightly longer than EP in OSA patients (AT $45 \pm 27 \mathrm{~s}$, EP $38 \pm 20 \mathrm{~s}$ for FIA, $P<0.05)$ indicating that a short struggle phase was present.

\section{Respiratory parameters}

Respiratory calculations are based on nine control subjects, due to one measurement failure. Baseline ${ }^{\mathrm{ET}} \mathrm{CO}_{2}$ did not differ between the groups or types of apneas (Table 2). All apneas caused ${ }^{\mathrm{ET}} \mathrm{CO}_{2}$ to rise $(P<0.001)$. In controls, FIA led to a smaller increase in ${ }^{\mathrm{ET}} \mathrm{CO}_{2}$ than $\mathrm{A}(P<0.01)$. This was not seen in the OSA group. In both types of apneas, controls increased their ${ }^{\mathrm{ET}} \mathrm{CO}_{2}$ more than did the OSA patients $(P<0.01$; Table 2$)$. Baseline $\mathrm{SaO}_{2}$ was the same in both groups. All apneas led to a decrease in $\mathrm{SaO}_{2}$ $(P<0.01)$. There was no significant difference in desaturation between $\mathrm{A}$ and FIA neither within any group nor between groups in any of the two conditions (A and FIA; Table 2).

\section{Diving response}

Baseline HR was the same in OSA and control subjects (NS). Both groups showed significant bradycardia during both types of apneas $(P<0.05$; Fig. 2). In controls, the mean HR reduction by the end of apnea was greater in FIA $(19 \pm 10 \%)$ than in A $(10 \pm 10 \% ; P<0.05)$ while among OSA it was similar in A and FIA $(13 \pm 10$ and $14 \pm 8 \%$; NS; Fig. 2). The more powerful response to FIA becomes evident in controls after approximately $25 \mathrm{~s}$ of apnea and continues in the early recovery. After apnea, both controls and OSA showed an increase in HR compared to baseline within $30 \mathrm{~s}$ in both types of apneas (Fig. 3). In this graph, the subjects who had the longest apneas were included.

Baseline SkBf differed between groups with a higher mean value for OSA patients $(P<0.05)$. In the OSA group,

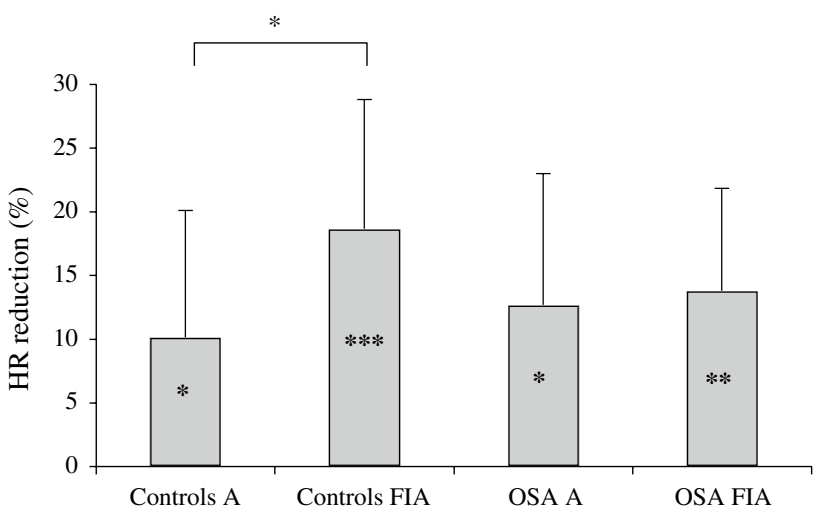

Fig. 2 Mean $( \pm S D)$ heart rate $(H R)$ reduction in control subjects $(n=10)$ and OSA patients $(n=8)$ during apnea (A) and apnea with face immersion (FIA). A significantly greater reduction in HR was noted in control subjects during FIA in comparison to apnea without face immersion. $* P \leq 0.05, * * P \leq 0.01, * * * P \leq 0.001$

SkBf was reduced by $15 \pm 14 \%(P<0.05)$ during A and by $29 \pm 23 \%(P<0.05)$ during FIA. No significant change from baseline was seen in controls. Baseline MAP was the same for both groups, and did not change significantly during apnea in any group or condition (NS).

\section{Hemoglobin concentration}

Hemoglobin values are based on nine control subjects and seven OSA patients, due to coagulation in two blood samples. There was no difference in baseline [Hb] between OSA and controls $(13.7 \pm 0.6$ vs. $13.3 \pm 1.0 \mathrm{~g} / \mathrm{dL} ; \mathrm{NS})$ or between A and FIA (NS). An increase in [Hb] was seen at $3 \mathrm{~min}$ after the apnea series in both groups during both A and FIA (Fig. 4). In controls, a tendency to an increase from baseline was seen in [Hb] by $2.2 \pm 2.9 \%$ after $\mathrm{A}$ $(P=0.056)$ and by $2.1 \pm 2.2 \%$ after FIA $(P<0.05)$, with no difference between values (NS). In the OSA group, A caused an increase from baseline of $1.4 \pm 0.9 \%(P<0.01)$ and FIA caused a $3.3 \pm 2.2 \%$ increase $(P<0.01)$, thus a more pronounced hematological response resulted when 

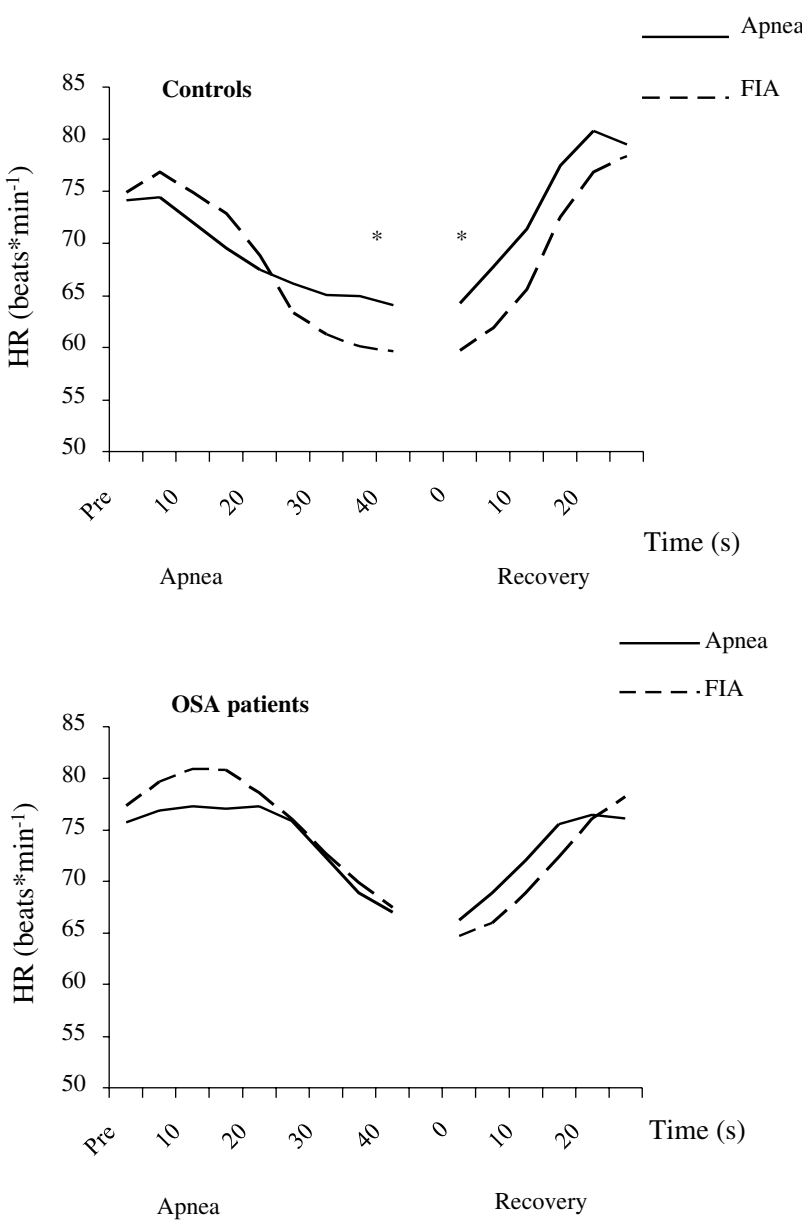

Fig. 3 Development of heart rate (HR) during apnea and the recovery phase in OSA patients $(n=5)$ and control subjects $(n=8)$ with apneas of at least $40 \mathrm{~s}$ duration. Values represent the mean value. Standard deviation is left out for clarity. A significant difference between A and FIA was seen in controls, both during apnea and during the recovery period. $* P \leq 0.05$

the face was immersed $(P<0.05)$. All increases were fully reversed within 20 min of recovery (Fig. 4).

\section{Discussion}

Both controls and OSA patients responded to apnea with a cardiovascular "diving response", in accordance with previous studies in healthy subjects (Elsner and Gooden 1983; Gooden 1994). In controls, the diving bradycardia was fortified by facial chilling, which has been reported in several previous studies (Andersson and Schagatay 1998a; de Bruijn et al. 2009). However, this typical fortification of the bradycardia was not seen in the OSA patients, who responded with a similar bradycardia in both conditions.

Instead, in the FIA condition for the OSA group, the elevation of $[\mathrm{Hb}]$ associated with the contraction of the spleen (Schagatay et al. 2001, 2005; Bakovic et al. 2003)
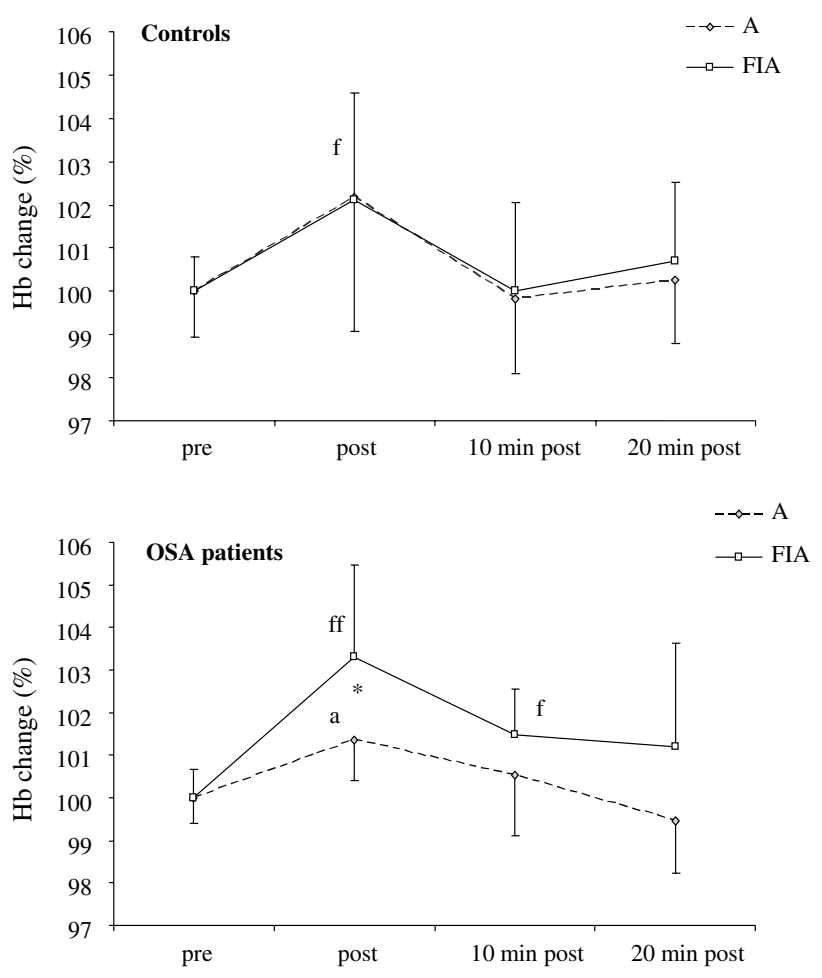

Fig. 4 Mean $( \pm S D)$ hemoglobin concentration in percent of resting value pre- and postapnea in OSA patients $(n=7)$ and in control subjects $(n=9)$. Resting value set as $100 \%$ in figure. Change from reference for A respective FIA at $P \leq 0.05$ is indicated by $a$ and $f$. Change from reference for A at $P \leq 0.01$ is indicated by $a a$ and for FIA by ff. Difference between A and FIA is indicated by asterisk for $P \leq 0.05$

was more pronounced than during apnea alone. A fortified $[\mathrm{Hb}]$ response in trained apnea divers compared to healthy non-divers and elite skiers during apnea with face immersion has previously been reported (Richardson et al. 2005), and OSA patients did show a similar elevation of the $[\mathrm{Hb}]$ response with face immersion. The reason for this difference between conditions is unclear.

The facial immersion induces a more powerful diving response via facial cold receptors in the ophthalmic region (Schuitema and Holm 1988), but it is unclear why this stimulation did not have any effect in the OSA group. Studies have found an increased sympathetic tone in OSA patients (Somers et al. 1995; Narkiewicz and Somers 2003). Diving bradycardia occurs as parasympathetic activity increases, affecting the cardiac pacemaker, and it is also likely that apnea induced reduction in sympathetic drive on the SA node is partly responsible for the diving bradycardia (Schagatay and Holm 1996; Paton et al. 2005). The increased resting sympathetic tone seen in OSA patients could possibly attenuate the reduction in heart rate normally seen when voluntary apneas are performed, and the reduction achieved in A could be the maximum achievable. The 
diving response has been shown to diminish in strength with increasing age, possibly due to structural changes in blood vessels (Kawasaki et al. 1987; Holm et al. 1998). Thus, even though our OSA patients were considered free from cardiovascular disease, this factor could be involved, since an increased incidence of vascular disease has been reported previously in this group (Wolf et al. 2007).

Arterial oxygen desaturation was seen in both groups after apneas, with and without face immersion. Previous studies on subjects performing 2 min breath-holds have shown an oxygen conserving effect of FIA associated with a stronger diving response (Andersson and Schagatay 1998a). This enhancement was present but did not reach significance in our study, probably due to the shorter apneic duration and subsequently lower degree of desaturation. It is likely that the more powerful diving response in FIA in the control group in the present study tended to reduce arterial desaturation, thereby diminishing an important stimulus for inducing spleen contraction and $[\mathrm{Hb}]$ elevation (Qvist et al. 1986; Richardson et al. 2009). This enhancement of the diving response was not seen in the OSA group, which could possibly explain their enhanced $[\mathrm{Hb}]$ response during FIA.

The magnitude of bradycardia is an easy and wellestablished measure of the magnitude of the overall diving response (Schagatay 1996; Schagatay and Andersson 1998; Andersson and Schagatay 1998a), but SkBF and MAP were also measured in this study. We found changes in SkBF between A and FIA in OSA but not in controls, and no changes in MAP in any group, thus the increase in total peripheral resistance apparently just matched the reduction in cardiac output in both groups. An increase of the MAP is often seen toward the end of the apnea, at least in studies involving longer apneas (Lin 1982), but in this study apneas were likely of to short duration for significant changes in MAP to develop.

In the present study, an elevation of [Hb] after apnea was found for both groups and the response was fortified after FIA in the OSA group suggesting a training effect, by frequent apneas, on spleen contraction in line with other studies involving long-term apnea training (Richardson et al. 2005; Engan et al. 2014). Diving bradycardia on the contrary, seemed to be somewhat attenuated in the OSA patients, with a slower onset and no augmentation from face immersion. A cause for this difference between the two responses may be that the increased resting sympathetic tone in the OSA group attenuates the cardiovascular response (i.e. the bradycardia) to apnea by not allowing a full sympathetic reduction to the beta receptors, while not affecting the spleen response which is mainly induced by a sympathetically derived release of catecholamines (Stewart and McKenzie 2002).
During apnea, rising $\mathrm{CO}_{2}$ and subsequent acidosis are likely the main ventilatory stimuli, but the sensitivity of the respiratory center to carbon dioxide is typically reduced in divers (Masuda et al. 1981; Davis et al. 1987; Lilly 1964). Some of the subjects in the OSA group performed short breath holding times and many terminated their apnea without a struggle phase, while in the control group a significant struggle phase was often seen (AT longer than EP) as a part of the total apnea time. This indicates a tendency to shorter breath holding time due to increased rather than decreased ventilatory drive in patients with OSA, as shown previously (Taskar et al. 1995; Appelberg and Sundstrom 1997) despite their frequent exposure to apneas.

Limitations of the study were the short apneic duration achieved in this group of elderly untrained subjects, the low number of subjects, and the unequal inclusion of males and females. However, in previous studies we have not found that the diving response differs between men and women, thus we expect no sex-linked influence on these parameters.

\section{Conclusions}

The response pattern during apnea was different in OSA patients compared to controls, but also different from the responses previously observed in trained apnea divers. Face immersion enhanced the apnea-induced diving response only in controls, while in the OSA group, the hemotological response was stronger when face-immersed apneas were performed. We concluded that OSA patients do not show a training-induced enhancement of the bradycardic response as a result of their nocturnal apneas but the $[\mathrm{Hb}]$ elevation, present during FIA, appears to be enhanced in the OSA subjects.

Acknowledgments We are grateful to all patients and control subjects for participating in the study. We also thank Dr. Matt Richardson, Dr. Sofia Pettersson, and M.Sc Torborg Jonsson, for technical assistance and B.A. Stephen Haughey for linguistic revision. This study was supported by Mid Sweden's Research and Development Center, Sundsvall Hospital, Landstinget Västernorrland and by Capio St Görans Hospital, Stockholm, Sweden.

Conflict of interest The authors have no conflicts of interest to disclose.

\section{References}

Andersson J, Schagatay E (1998a) Arterial oxygen desaturation during apnoea in humans. Undersea Hyperb Med 25:21-25

Andersson J, Schagatay E (1998b) Effects of lung volume and involuntary breathing movements on human diving response. Eur $\mathrm{J}$ Appl Physiol 77:19-24 
Appelberg J, Sundstrom G (1997) Ventilatory response to $\mathrm{CO}_{2}$ in patients with snoring, obstructive hypopnoea and obstructive apnoea. Clin Physiol 17:497-507

Bakovic D, Valic Z, Eterovic D, Vukovic I, Obad A, Marinovic-Terzic I, Dujic Z (2003) Spleen volume and blood flow response to repeated breath-hold apnoeas. J Appl Physiol 95:1460-1466

Butler PJ, Jones DR (1997) Physiology of diving of birds and mammals. Physiol Rev 77:837-899

Daly MD, Hazzledine JL (1963) The effects of artificially induced hyperventilation on the primary cardiac reflex response to stimulation of the carotid bodies in the dog. J Physiol 168:872-889

Davis FM, Graves MP, Guy HJ, Prisk GK, Tanner TE (1987) Carbon dioxide response and breath-hold times in underwater hockey players. Undersea Biomed Res 14:527-534

de Bruijn R, Richardson MX, Schagatay E (2009) Oxygen-conserving effect of the diving response in the immersed human. Diving Hyperb Med 39:193-199

Dejours P (1965) Hazards of hypoxia during diving. In: Rahn H, Yokoyama T (eds) Physiology of breath-hold diving and the Ama of Japan. National Academy of Science, National Research Council, Washington DC, pp 183-193

Dingli K, Coleman EL, Vennelle M, Finch SP, Wraith PK, Mackay TW, Douglas NJ (2003) Evaluation of a portable device for diagnosing the sleep apnoea/hypopnoea syndrome. Eur Respir J 21:253-259

Elsner R, Gooden B (1983) Diving and asphyxia : a comparative study of animals and man. Monogr Physiol Soc 40:1-168

Engan H, Richardson M, Lodin Sundström A, van Beekvelt M, Schagatay E (2013) Effects of 2 weeks of daily apnea training on diving response, spleen contraction, and erythropoiesis in novel subjects. Scand J Med Sci Sports 23:340-348

Engan H, Lodin-Sundström A, Schagatay F, Schagatay E (2014) The effect of climbing Mount Everest on spleen contraction and increase in hemoglobin concentration during breath holding and exercise. High Altitude Med Biol 15:52-57

Gooden BA (1994) Mechanism of the human diving response. Integr Physiol Behav Sci 29:6-16

Holm B, Schagatay E, Kobayashi T, Masuda A, Ohdaira T, Honda Y (1998) Cardiovascular change in elderly male breath-hold divers (Ama) and their socio-economical background at Chikura in Japan. Appl Human Sci 17:181-187

Hurford WE, Hong SK, Park YS, Ahn DW, Shiraki K, Mohri M, Zapol WM (1990) Splenic contraction during breath-hold diving in the Korean ama. J Appl Physiol 69:932-936

Kawasaki T, Sasayama S, Yagi S, Asakawa T, Hirai T (1987) Non-invasive assessment of the age related changes in stiffness of major branches of the human arteries. Cardiovasc Res $21: 678-687$

Lilly JC (1964) Animals in aquatic environments: Adaptation of mammals to the ocean. In: Dill DB, Adolph EF (eds) Handbook of physiology: a critical comprehensive presentation of physiological knowledge and concepts-Section 4: adaption to the environment. American Physiological Society, Bethesda, pp 714-747

Lin YC (1982) Breath-hold diving in terrestrial mammals. Exerc Sport Sci Rev 10:270-307

Lin YC, Lally DA, Moore TO, Hong SK (1974) Physiological and conventional breath-hold breaking points. J Appl Physiol 37:291-296

Lodin-Sundstrom A, Schagatay E (2010) Spleen contraction during 20 min normobaric hypoxia and 2 min apnoea in humans. Aviat Space Environ Med 81:545-549

Masuda Y, Yoshida A, Hayashi F, Sasaki K, Honda Y (1981) The ventilatory responses to hypoxia and hypercapnia in the ama. Jpn J Physiol 31:187-197
Narkiewicz K, Somers VK (2003) Sympathetic nerve activity in obstructive sleep apnoea. Acta Physiol Scand 177:385-390

Palada I, Bakovic D, Valic Z, Obad A, Ivancev V, Eterovic D, Shoemaker JK, Dujic Z (2008) Restoration of hemodynamics in apnoea struggle phase in association with involuntary breathing movements. Respir Physiol Neurobiol 161:174-181

Paton JF, Boscan P, Pickering AE, Nalivaiko E (2005) The yin and yang of cardiac autonomic control: vago-sympathetic interactions revisited. Brain Res Brain Res Rev 49:555-565

Qvist J, Hill RD, Schneider RC, Falke KJ, Liggins GC, Guppy M, Elliot RL, Hochachka PW, Zapol WM (1986) Hemoglobin concentrations and blood gas tensions of free-diving Weddell seals. $\mathbf{J}$ Appl Physiol 61:1560-1569

Richardson M, de Bruijn R, Holmberg HC, Bjorklund G, Haughey H, Schagatay E (2005) Increase of hemoglobin concentration after maximal apnoeas in divers, skiers, and untrained humans. Can J Appl Physiol 30:276-281

Richardson MX, de Bruijn R, Schagatay E (2009) Hypoxia augments apnoea-induced increase in hemoglobin concentration and hematocrit. Eur J Appl Physiol 105:63-68

Schagatay E (1996) The human diving response: effects of temperature and training (Thesis), Department of Animal Physiology, University of Lund, Lund

Schagatay E (2009) Review: predicting performance in competitive apnoea diving, part I: static apnoea. Div Hyperb Med 39(2):88-99

Schagatay E, Andersson J (1998) Diving response and apneic time in humans. Undersea Hyperb Med 25:13-19

Schagatay E, Haughey H, Reimers J (2005) Speed of spleen volume changes evoked by serial apnoeas. Eur J Appl Physiol 93:447-452

Schagatay E, Holm B (1996) Effects of water and ambient air temperatures on human diving bradycardia. Eur J Appl Physiol Occup Physiol 73:1-6

Schagatay E, van Kampen M, Emanuelsson S, Holm B (2000) Effects of physical and apnoea training on apneic time and the diving response in humans. Eur J Appl Physiol 82:161-169

Schagatay E, Andersson JPA, Hallen M, Palsson B (2001) Selected contribution: role of spleen emptying in prolonging apnoeas in humans. J Appl Physiol 90:1623-1629 (discussion 1606)

Schagatay E, Andersson JPA, Nielsen B (2007) Hematological response and diving response during apnoea and apnoea with face immersion. Eur J Appl Physiol 101:125-132

Schuitema K, Holm B (1988) The role of different facial areas in eliciting human diving bradycardia. Acta Physiol Scand 132:119-120

Somers VK, Dyken ME, Clary MP, Abboud FM (1995) Sympathetic neural mechanisms in obstructive sleep apnoea. J Clin Invest 96:1897-1904

Stewart IB, McKenzie DC (2002) The human spleen during physiological stress. Sports Med 32:361-369

Taskar V, Clayton N, Atkins M, Shaheen Z, Stone P, Woodcock A (1995) Breath-holding time in normal subjects, snorers, and sleep apnoea patients. Chest 107:959-962

Tolle FA, Judy WV, Yu PL, Markand ON (1983) Reduced stroke volume related to pleural pressure in obstructive sleep apnoea. $\mathrm{J}$ Appl Physiol 55:1718-1724

Wolf J, Lewicka J, Narkiewicz K (2007) Obstructive sleep apnoea: an update on mechanisms and cardiovascular consequences. Nutr Metab Cardiovasc Dis 17:233-240

Zwillich C, Devlin T, White D, Douglas N, Weil J, Martin R (1982) Bradycardia during sleep apnoea. Characteristics and mechanism. J Clin Invest 69:1286-1292 